\title{
DESENVOLVIMENTO DE NANOEMULSÕES POLIMÉRICAS CATIÔNICAS PARA LIBERAÇÃO CONTROLADA DE CURCUMINÓIDES FOTOSSENSIBILIZADORES DE ORIGEM BIOTECNOLÓGICA
}

\author{
L.G. MUNHOZ ${ }^{1}$, C.F. AMANTINO ${ }^{1}$, A.C. TEDESCO ${ }^{2}$ e F.L. PRIMO ${ }^{1 *}$ \\ ${ }^{1}$ Faculdade de Ciências Farmacêuticas de Araraquara, Departamento de Bioprocessos e \\ Biotecnologia, Universidade Estadual Paulista Júlio de Mesquita Filho - UNESP. \\ ${ }^{2}$ Departamento de Química, FFCLRP, Universidade de São Paulo - USP \\ luciana.gmunhoz@gmail.com; flprimo@fcfar.unesp.br*
}

\begin{abstract}
RESUMO - O uso incorreto e desorientado de antibióticos tem tido como consequência o aparecimento de microrganismos mais resistentes aos tratamentos convencionais. $\mathrm{O}$ uso da fotoinativação microbiana junto com a nanotecnologia tem surgido como uma alternativa de tratamento para esses e outros casos. A fotoinativação consiste na utilização de um fotossenssibilizador combinando-se a aplicação controlada de irradiação de luz monocromática, que desencadeia numa série de reações que causam a morte do microrganismo. A nanoencapsulação desses ativos fotossensibilizadores pode resultar na melhoria das características físico-químicas destes compostos, como o aumento de estabilidade, redução da fotodegradação, aumento da área superficial de interação, biocompatibilidade, além de também preservar e potencializar as características dos biocompostos. Neste contexto o presente trabalho tem como objetivo o desenvolvimento e caracterização de compostos nanoestruturados contendo a curcumina como ativo fotossensibilizante, para futura aplicação em inativação microbiana empregandose processos fotodinâmicos.
\end{abstract}

\section{INTRODUÇÃO}

\subsection{Curcumina}

Como Sueth-Santiago et al. (2015) demonstrou, a curcumina é um composto de origem natural, sendo o rizoma encontrado em um arbusto que é denominado cúrcuma longa. A curcumina despertou o interesse de pesquisadores devido as suas propriedades farmacológicas como por exemplo, atividade anti-inflamatória, antimicrobiana, cicatrizante e digestiva. A estrutura química desse composto baseia-se em dois anéis fenólicos e duas cetonas, como pode ser observado na Figura 1. 
Figura 1 - Estrutura química da curcumina<smiles>COc1cc(/C=C/C(=O)CC(=O)/C=C/c2ccc(O)c(OC)c2)ccc1O</smiles>

Segundo Suzuki (2016), sua estrutura química e atividades biológicas são características de interesse para a aplicação na terapia fotodinâmica, pois absorvem preferencialmente na faixa de 400-430 nm. Ainda no trabalho de Suzuki (2016) percebe-se que a natureza hidrofóbica da curcumina é uma limitação para sua aplicação em meio biológico. Devido a sua baixa solubilidade em solução aquosa, o uso da nanotecnologia auxilia no aumento da sua solubilidade, biodistribuição e biodisponibilidade no meio biológico.

\subsection{Nanotecnologia}

Conforme referido no trabalho de Primo (2011), a nanotecnologia contribui diretamente para o desenvolvimento de novos materiais, processos e dispositivos para diversas aplicações, destacando-se nas áreas da saúde humana e a biotecnológica. Os princípios da nanotecnologia são fundamentados no desenvolvimento de novos materiais em escalas nanométricas. Chaudhry et al. (2010) mostra que os materiais nanoestruturados possuem potencial diferenciado frente a outros materiais em escala de micro e/ou macroscópica, podendo ter propriedades físico-químicas potencializadas, resultando em características únicas e propriedades diferentes das observadas em outras escalas de tamanhos superiores. Segundo Weiss et al (2006) dentre os diferentes tipos de nanomateriais estão as nanocápsulas, nanoemulsões, nanolaminados, nanotubos e nanofibras.

De acordo com Perez et al (2012) as nanoemulsões (NE) destacam-se devido sua estabilidade físico-química ampliada, podendo ser obtidas por processos de emulsificação espontânea e deposição polimérica interfacial pela combinação de co-polímeros e diferentes óleos.

As NE promovem o aumento da biodisponibilidade dos ativos incorporados em sua matriz coloidal, resultando na alteração da digestão e metabolismo desses ativos pelas presenças dos lipídios que compõe a nanoemulsão, além de também influenciar no aumento do "tempo de prateleira" dessas formulações, visto que as protegem de fenômenos físico-químicos degradantes como a volatilização e oxidação, que comumente levam a instabilidade do medicamento e a redução do seu prazo de validade, como mostrado por Jeong (2007).

\section{OBJETIVOS}

O trabalho em questão tem como principal objetivo, desenvolver e caracterizar compostos fotossensibilizadores nanoestruturados que sejam de origem natural e/ou obtidos por via biotecnológica. 


\section{MÉTODOS}

As nanoemulsões catiônicas (NE) foram preparadas segundo o método de emulsificação espontânea interfacial apresentado por Siqueira-Moura et al 2012. Inicialmente foram preparadas duas soluções: uma fase aquosa contendo o polímero Kolliphor P188 (Sigma-Aldrich Co.) a 7,5 \% em água ultrapura, e uma fase orgânica com acetona, contendo Lipoid S100 (Lipoid AG) a 7,5\% a $55^{\circ} \mathrm{C}$. Para alterar a carga residual final foi adicionado o polímero catiônico brometo de didodecildimetilamônio - DDAB (Sigma-Aldrich Co.), para modulação da carga superficial desejada. Foram testadas diferentes frações de DDAB (7,8 a $31 \mathrm{mg} / \mathrm{mL}$ ) buscando a melhor curva de estabilidade físico química das formulações. $\mathrm{O}$ fotossensibilizador curcumina (Sigma-Aldrich Co.) foi adicionado à fase orgânica após dissolução em óleo Edenor KS (Cosmoquímica Ltda.), previamente a etapa de pré-emulsificação, para uma concentração final de $0,1 \mathrm{mg} / \mathrm{mL}$. A formulação final foi obtida por emulsificação a pressão reduzida após remoção de todo solvente orgânico numa velocidade de rotação de $125 \mathrm{rpm}$ e à temperatura de $55{ }^{\circ} \mathrm{C}$. Para caracterização dos nanomateriais obtidos foi empregada a técnica de espalhamento dinâmico de luz (DLS dynamic light scattering), para determinação do tamanho de partícula, potencial Zeta $(\zeta)$ e índice de polidispersão (PdI), utilizando-se um equipamento Zetasizer, modelo ZS90 da Malvern Instruments. Estas análises foram realizadas em colaboração com os Profs. Dr. Miguel Jafelicci Júnior e Dr. Rodrigo Fernando Costa Marques, do Departamento de FísicoQuímica do Instituto de Química da UNESP-Araraquara.

\section{RESULTADOS E DISCUSSÃO.}

Foram desenvolvidas nanoemulsões com três frações diferentes do polímero catiônico. A Tabela 1 mostra os dados de tamanho, índice de polidispersão e potencial zeta das nanoemulsões em função do tempo.

Tabela 1 - Dados de tamanho de partícula, índice de polidispersão (PdI) e potencial Zeta $(\zeta)$, em função da concentração do DDAB

\begin{tabular}{|c|c|c|c|c|c|c|c|c|c|}
\hline \multirow[b]{2}{*}{ Dias } & \multicolumn{3}{|c|}{ Concentração $31 \mathrm{mg} / \mathrm{ml}$} & \multicolumn{3}{|c|}{ Concentração 15,64 mg/ml } & \multicolumn{3}{|c|}{ Concentração $7,82 \mathrm{~g} / \mathrm{ml}$} \\
\hline & Tamanho $(\mathrm{nm})$ & Potencial (mV) & Pdl & Tamanho (nm) & Potencial (mV) & Pdl & Tamanho (nm) & Potencial (mV) & Pdl \\
\hline 1 & 486 & 84,5 & 0,33 & 183,5 & 76,8 & 0,20 & 246,0 & 72,0 & 0,29 \\
\hline 8 & 478 & 91,3 & 0,30 & 186,6 & 92,5 & 0,20 & 269,3 & 67,5 & 0,28 \\
\hline 12 & 711 & 95,3 & 0,43 & 194,8 & 87,0 & 0,18 & 227,4 & 106,0 & 0,25 \\
\hline 25 & 471 & 75,7 & 0,30 & 195,0 & 85,6 & 0,16 & 248,7 & 77,6 & 0,33 \\
\hline 34 & 429 & 92,9 & 0,35 & 183,8 & 84,2 & 0,18 & 214,6 & 89,6 & 0,38 \\
\hline
\end{tabular}

Os resultados apresentados na Tabela 1 demonstram o perfil de estabilidade para as formulações NEC contendo diferentes concentrações de DDAB. Os dados indicam uma estabilidade físico-química para os primeiros 34 dias de análise, entretanto as amostras com concentração de DDAB $=15,64 \mathrm{mg} / \mathrm{mL}$ apresentaram um tamanho de partícula reduzido (< $200 \mathrm{~nm}$ ). Os potenciais Zeta foram obtidos numa faixa de valor $>80 \mathrm{mV}$ para as NEC de preparadas com as concentrações de $15,64 \mathrm{mg} / \mathrm{mL}$ e $31 \mathrm{mg} / \mathrm{mL}$ de DDAB. Após o tratamento dos dados foi definido que a nanoemulsão contendo a concentração de $15,64 \mathrm{mg} / \mathrm{mL}$ de DDAB catiônico foi a que apresentou melhor perfil de estabilidade $(\mathrm{d}<200 \mathrm{~nm}, \zeta>80 \mathrm{mV}$ e PdI < 0,2). Desta forma, foi definido que uma nanoemulsão encapsulando o ativo fotossensibilizador curcumina, fosse preparada contendo esta proporção polimérica. Na 


\section{Congresso Brasileiro de Engenharia Química em Iniciação Científica UFSCar - São Carlos - SP 16 a 19 de Julho de 2017}

Tabela 2 temos os dados de caracterização físico-química para a NEC contendo a curcumina nanoencapsulada.

Tabela 2 - Dados de tamanho de partícula, índice de polidispersão e potencial zeta da nanoemulsão catiônica com curcumina

\begin{tabular}{|c|c|c|c|}
\hline & \multicolumn{3}{|c|}{ Concentração 15,64 mg/ml } \\
\hline Dias & Tamanho $(\mathrm{nm})$ & Potencial $(\mathrm{mV})$ & Pdl \\
\hline 1 & 155,3 & 89,8 & 0,21 \\
\hline 14 & 169,0 & 76,9 & 0,19 \\
\hline 23 & 175,5 & 104,0 & 0,16 \\
\hline
\end{tabular}

Os resultados obtidos na Tabela 2 demonstram que o perfil de tamanho de partícula, $\zeta$ e PDI foram mantidos após o encapsulamento do ativo fotossensibilizador. Na etapa seguinte dos estudos de caracterização físico-química do nanomaterial, foram realizadas análises de excitação, emissão de fluorescência da NEC contendo a curcumina, como apresentado nas Figura 2, 3 e 4 a seguir.

Figura 2 - Espectro de fluorescência da curcumina, medido com Spectrum type 3D, velocidade de escaneamento $2000 \mathrm{~nm} / \mathrm{min}$

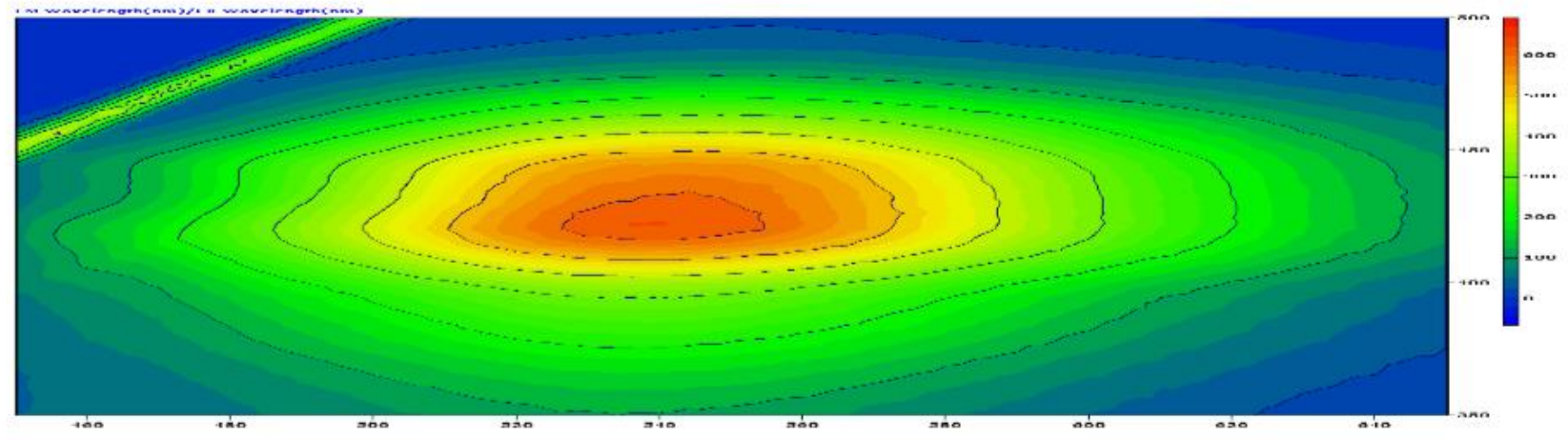

Figura 3 - Emissão da nanoemulsão catiônica medido por Spectrum type 3D, velocidade de escaneamento $2000 \mathrm{~nm} / \mathrm{min}$. $\lambda$ excitação $=378 \mathrm{~nm}$




Figura 4 - Excitação da nanoemulsão catiônica de curcumina medido por Spectrum type 3D, velocidade de escaneamento $2000 \mathrm{~nm} / \mathrm{min}, \lambda$ excitação $=378 \mathrm{~nm}$
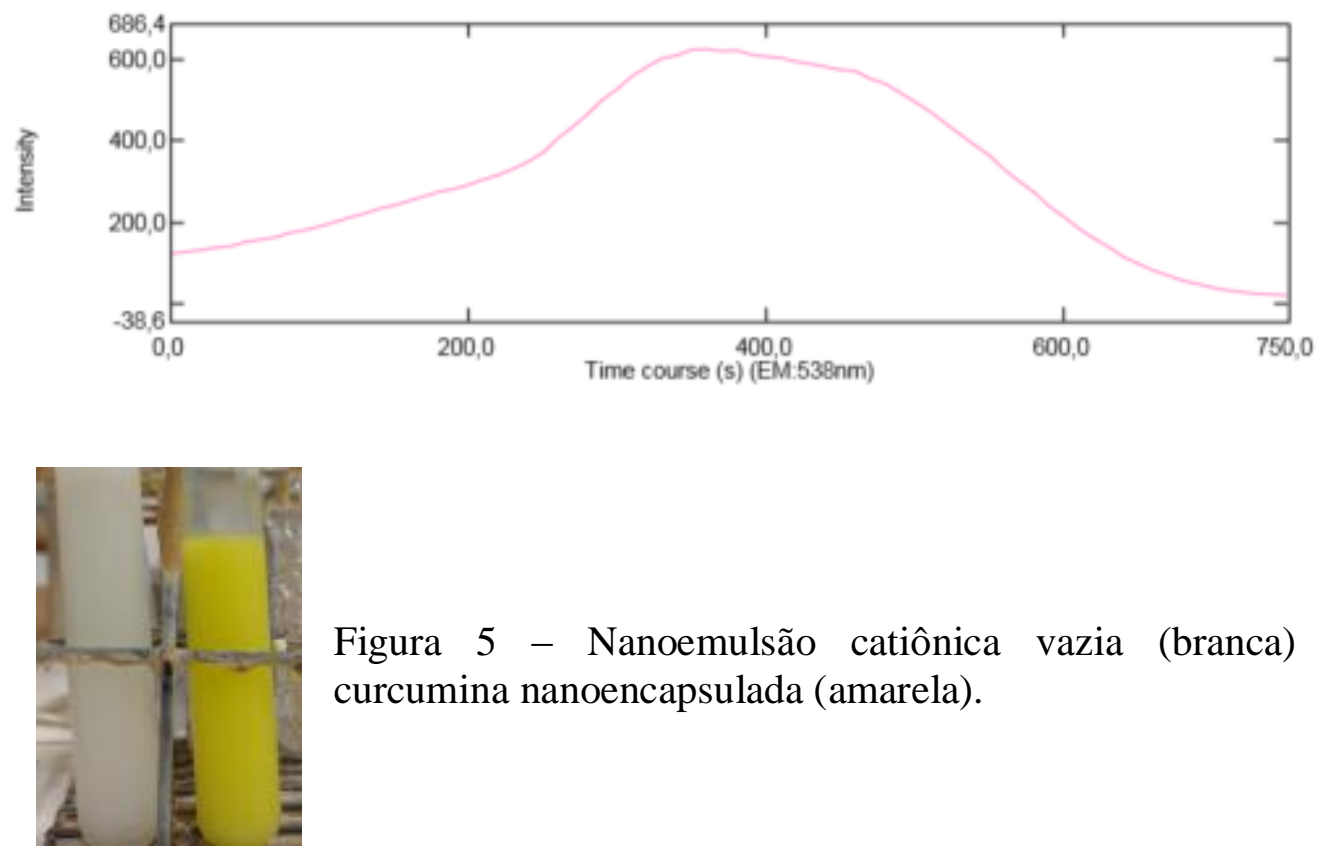

Figura 5 - Nanoemulsão catiônica vazia (branca) e com curcumina nanoencapsulada (amarela).

\section{CONCLUSÃO}

Concluímos que foi possível obter uma NEC para liberação controlada de derivados curcuminóides, a qual possui características físico-químicas adequadas conforme esperado para sistemas coloidais nanocarreadores. Com base nestes resultados preliminares é possível atestar que a formulação em questão tem potencial para utilização em protocolos de fotoinativação microbiana baseada em Processos Fotodinâmicos.

Agradecimentos: Os autores agradecem pela bolsa de iniciação científica concedida pelo Edital Primeiros Projetos/PROPe/UNESP, 09/2016 - Processo 765, 2016 - vigente (L.G.M). Agradecemos também ao Prof. Dr. Jorge Fernando Brandão Pereira, do Departamento de Bioprocessos e Biotecnologia da Faculdade de Ciências Farmacêuticas de Araraquara - UNESP, por ceder o equipamento espectrofluorímetro Spectrum 3D para as análises de excitação e emissão de fluorescência das formulações e aos Profs. Dr. Miguel Jafelicci Júnior e Dr. Rodrigo Fernando Costa Marques, do Departamento de Físico-Química do Instituto de Química da UNESP-Araraquara, por ceder o equipamento Zetasizer para análises de tamanho.

\section{REFERÊNCIAS}

CHAUDHARY, J. H; BANO, A.S.W; ULLAH,F; MUNIS, F; FAHAD, S; AHMAD, I. In vitro analysis of Cupressus sempervirens L. plant extracts antibaterial activity. Journal of Medicinal Plants Research, V. 6, p. 273-276, 2012. 
JEONG, S. H.; PARK, J. H.; PARK, K. Formulation issues around lipid-based oral and parenteral delivery systems: role of lipid excipients in modifying oral and parenteral drug delivery. Hoboken: Wiley-Interscience, 2007

PEREZ, S.,F; BERTAGNOLLI, M.M; ALVES, P. M; PENNA, G.N. Nanotecnologia: aplicação na área de alimentos, Disc.Scientia, v. 13, p. 97-110, 2012.

PRIMO, F. L.; REIS, M. B. D.; PORCIONATTO, M. A.; TEDESCO, A. C. In Vitro Evaluation of Chloroaluminum Phthalocyanine Nanoemulsion and Low-Level Laser Therapy on Human Skin Dermal Equivalents and Bone Marrow Mesenchymal Stem Cells, Current Medicinal Chemistry, v. 18, p. 3376-3381, 2011.

ROSA, L.L; BRUXEL, F; BIDONE, J; TEIXEIRA, F.H. Efeito da concentração de fase oleosa no tamanho de gotícula de nanoemulsões para terapia gênica. Anais do salão internacional de ensino pesquisa e extensão, v.7, 2015.

SANTOS, F.D.P. Avaliação da atividade antimicrobiana de microcristais de curcumina, Universidade tecnológica federal do paraná,2015.

SIQUEIRA-MOURA, M.P.; BOLFARINI， C. G.; DEMETS, F.J.G; TEDESCO, C.A.Preparation, characterization, and in vitro phototoxic effect of zinc phthalocyanine cucurbit[7]uril complex encapsulated into liposomes. Dyes and Pigments, p.162-167, vol.100, 2014.

SUETH-SANTIAGOA, V; MENDES-SILVA, P. G.; DECOTÉ-RICARDO, D.; LIMA, F. E.M. curcumina, o pó dourado do açafrão-da-terra: introspecções sobre química e atividades biológicas. Quim. Nova, v. 38, p. 538-552, 2015

SUZUKI, L.I. Viabilização da curcmina natural nanoencapsulada para inativação fotodinâmica. Instituto de física de São Carlos, 2016

WEISS, J; TAKHISTOV,J; MCCLEMENTS, J. D. Functional materials in food nanotecnology, Journal of food Science, v. 7, p. 107 - 116, 2006. 